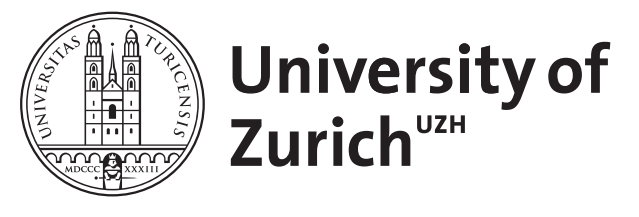

\title{
CT und MRT der Hüftprothese
}

\author{
Agten, C A ; Sutter, R ; Pfirrmann, C W A
}

\begin{abstract}
Metal-induced artifacts impair image quality of computed tomography (CT) and magnetic resonance imaging $(\mathrm{MRI})$ in patients with hip prostheses. Due to new developments in metal artifact reduction both methods can now be used for evaluation of a painful hip prosthesis. Iterative reconstruction algorithms and dual-energy scans are among the newer CT techniques for artifact reduction, while slice-encoding for metal artifact correction (SEMAC) and multi-acquisition variable-resonance image combination (MAVRIC) have introduced substantial improvements for MRI. Loosening of the hip prosthesis, osteolysis from small wear particles and pseudotumors in metal-on-metal prostheses are specific pathologies in patients with total hip arthroplasty. Other causes of painful hip prostheses are infections, fractures, tendinopathies, tendon ruptures, muscle and nerve alterations and heterotopic ossifications.
\end{abstract}

DOI: https://doi.org/10.1007/s00117-014-2693-8

Other titles: CT and MRI of hip arthroplasty

Posted at the Zurich Open Repository and Archive, University of Zurich

ZORA URL: https://doi.org/10.5167/uzh-106087

Journal Article

Published Version

Originally published at:

Agten, C A; Sutter, R; Pfirrmann, C W A (2014). CT und MRT der Hüftprothese. Der Radiologe, 54(7):715-725; quiz 726.

DOI: https://doi.org/10.1007/s00117-014-2693-8 


\section{Rubrikherausgeber}

S. Delorme, Heidelberg (Leitung)

P. Reimer, Karlsruhe

W. Reith, Homburg/Saar

C. Schäfer-Prokop, Amersfoort

C. Schüller-Weidekamm, Wien

M. Uhl, Freiburg

\section{Punkte sammeln auf: \\ springermedizin.de/ \\ eAkademie \\ Teilnahmemöglichkeiten \\ Diese Fortbildungseinheit steht Ihnen als e.CME und e.Tutorial in der Springer Medizin e.Akademie zur Verfügung. \\ - e.CME: kostenfreie Teilnahme im Rahmen des jeweiligen Zeitschriften- abonnements \\ - e.Tutorial:Teilnahme im Rahmen des e.Med-Abonnements}

\section{Zertifizierung}

Diese Fortbildungseinheit ist mit 3 CMEPunkten zertifiziert von der Landesärztekammer Hessen und der Nordrheinischen Akademie für Ärztliche Fort- und Weiterbildung und damit auch für andere Ärztekammern anerkennungsfähig.

\section{Hinweis für Leser aus Österreich} Gemäß dem Diplom-Fortbildungs-Programm (DFP) der Österreichischen Ärztekammer werden die in der e.Akademie erworbenen CME-Punkte hierfür 1:1 als fachspezifische Fortbildung anerkannt.

\section{Kontakt und weitere}

Informationen

Springer-Verlag GmbH

Springer Medizin Kundenservice

Tel. 08007780777

E-Mail:kundenservice@springermedizin.de

\section{CME Zertifizierte Fortbildung}

\author{
C.A. Agten $\cdot$ R. Sutter $\cdot$ C.W.A. Pfirrmann
}

Radiologie, Uniklinik Balgrist, Zürich

\section{CT und MRT der Hüftprothese}

\section{Zusammenfassung}

Metallartefakte erschweren die Beurteilung von Computer- (CT) und Magnetresonanztomographie (MRT) bei Patienten mit Hüftprothesen. Neuere technische Entwicklungen erlauben heute, beide Methoden bei Patienten mit schmerzhaften Hüftprothesen mit Erfolg einzusetzen. Neuere Technologien zur Metallartefaktreduktion in der CT sind unter anderem iterative Rekonstruktionsalgorithmen und Dual-Energy-CT, in der MRT neuere Sequenzen wie SEMAC („slice-encoding for metal artifact correction“) oder MAVRIC („multi-acquisition variable-resonance image combination"). Lockerung der Hüftprothese, Osteolysen durch Abriebpartikel und Pseudotumoren bei Metall-Metall-Prothesen sind wichtige Krankheitsbilder bei Hüftprothesen. Infektionen, Frakturen, Tendinopathien, Sehnenrupturen, Muskelund Nervenschäden sowie heterotope Ossifikationen sind weitere Ursachen einer schmerzhaften Hüftprothese.

\section{Schlüsselwörter}

Computertomographie $\cdot$ Magnetresonanztomographie $\cdot$ Metallartefaktreduktion ·

Hüftgelenkersatz · Metall-Metall-Prothese 


\section{Lernziele}

Nach Lektüre dieses Beitrags ...

- verstehen Sie die Entstehung von Metallartefakten in CT und MRT.

- kennen Sie bisherige und neuere Methoden zur Metallartefaktreduktion in CT und MRT.

- sind Sie mit periprothetischen Osteolysen, Pseudotumoren und der Problematik der Metall-Metall-Prothesen vertraut.

— haben Sie einen Überblick über wichtige Differenzialdiagnosen bei Patienten mit einer schmerzhaften Hüftprothese.

\section{Einführung}

Sowohl bei der MRT als auch bei der CT sind Metallartefakte durch die Prothese ein erheblicher Störfaktor

Aufhärtungsartefakte entstehen durch zunehmende Aufhärtung von Röntgenstrahlen beim Durchtritt durch den Patienten
Die Ursachen einer schmerzhaften Hüftprothese sind vielfältig. Die initiale Abklärung nach Anamnese und klinischer Untersuchung erfolgt mittels konventionellem Röntgenbild. Bei Unklarheiten helfen die Computertomographie (CT) oder die Magnetresonanztomographie (MRT) weiter. Bei beiden Schnittbildverfahren sind Metallartefakte durch die Prothese ein erheblicher Störfaktor. In den letzten Jahren wurden aber bei der Metallartefaktreduktion für die CT und die MRT deutliche technische Fortschritte erzielt, sodass beide Methoden heute zur weiteren Abklärung von schmerzhaften Hüftprothesen eingesetzt werden können.

\section{Technik}

\section{Computertomographie}

\section{Entstehung von Metallartefakten}

Aufhärtungsartefakte entstehen durch zunehmende Aufhärtung von Röntgenstrahlen beim Durchtritt durch den Patienten. Die Röntgenstrahlen bei einer normalen CT sind polychromatisch, besitzen also ein breites Energiespektrum. Beim Durchtritt durch den Körper werden niederenergetische Photonen stärker absorbiert als höherenergetische Photonen. Die mittlere Photonenenergie nimmt dadurch zu, was bei Metallimplantaten je nach Projektion zu Streifenartefakten unterschiedlicher Stärke führt [1].

„Photon-starvation“-Artefakte entstehen, wenn durch die starke Strahlenabsorption durch Metall nur noch wenige Photonen am Detektor ankommen. Das führt zu einem verstärkten Bildrauschen mit streifigem Aspekt. Klassischerweise sieht man diesen Effekt im CT-Thorax auf Schulterhöhe oder im Becken $[2,3]$.

\section{CT and MRI of hip arthroplasty}

\section{Abstract}

Metal-induced artifacts impair image quality of computed tomography (CT) and magnetic resonance imaging (MRI) in patients with hip prostheses. Due to new developments in metal artifact reduction both methods can now be used for evaluation of a painful hip prosthesis. Iterative reconstruction algorithms and dual-energy scans are among the newer CT techniques for artifact reduction, while slice-encoding for metal artifact correction (SEMAC) and multi-acquisition variable-resonance image combination (MAVRIC) have introduced substantial improvements for MRI. Loosening of the hip prosthesis, osteolysis from small wear particles and pseudotumors in metal-on-metal prostheses are specific pathologies in patients with total hip arthroplasty. Other causes of painful hip prostheses are infections, fractures, tendinopathies, tendon ruptures, muscle and nerve alterations and heterotopic ossifications.

\section{Keywords}

Computed tomography $\cdot$ Magnetic resonance imaging $\cdot$ Metal artifact reduction $\cdot$ Hip replacement · Metal-on-metal prosthesis 


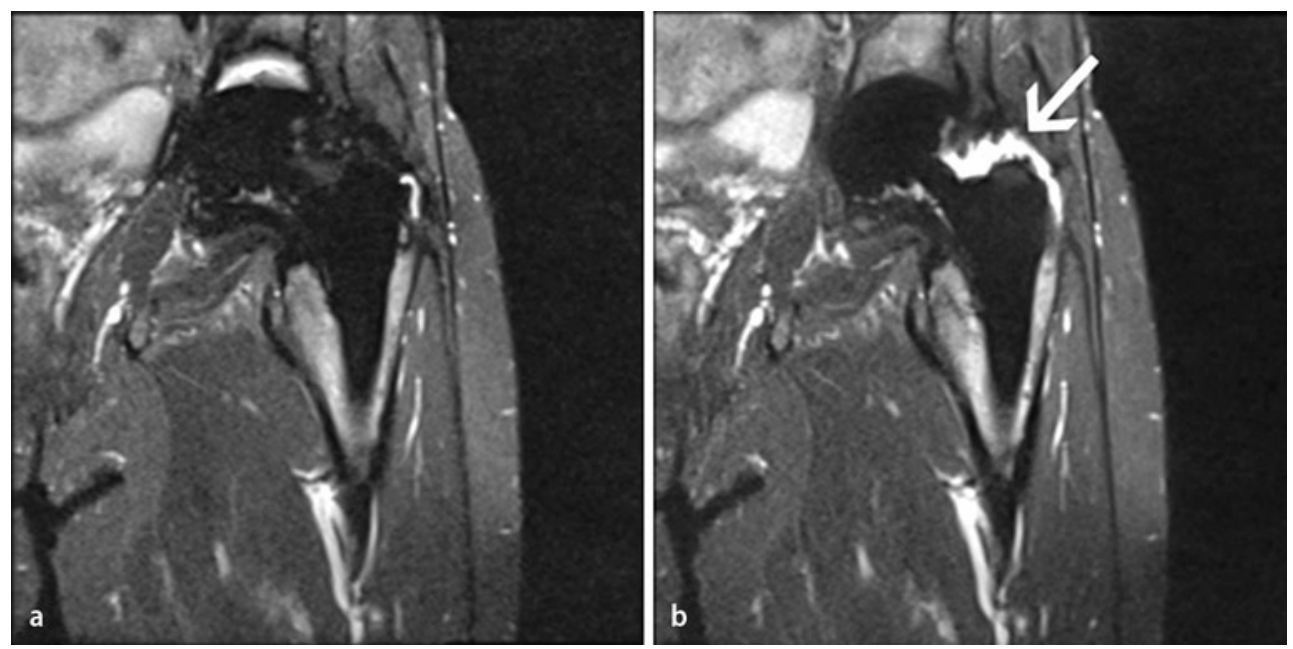

Abb. $1 \Delta$ Magnetresonanztomographie des linken Hüftgelenks bei Patient mit Hüftprothese in koronaler Ebene: a STIR mit hoher Empfängerbandbreite; b STIR mit SEMAC („slice-encoding for metal artifact correction"). Die Artefaktgröße um die Hüftprothese hat mit SEMAC deutlich abgenommen. In (b) zeigt sich jetzt eine Flüssigkeitskollektion (weißer Pfeil) um den Trochanter major bei Ablösung der Gluteus-medius-Sehne

"Scatter"-Artefakte entstehen durch Streuung von Photonen. Diese ändern im Patienten ihre Richtung und ihre Energie und landen auf einem anderen Detektorelement, was zu entsprechendem Rauschen führt [4].

Partialvolumeneffekte kommen v. a. bei breiter Kollimation oder exzentrischer Lage des Metalls im Körper zustande. Im zweiten Fall liegt das dichte Objekt nicht aus jeder Richtung im Röntgenstrahl der rotierenden CT-Röhre.

Die Artefakte sind v. a. in unmittelbarer Metallnähe am stärksten ausgeprägt. Das führt dazu, dass das Metall-Knochen- oder Metall-Weichteil-Interface in der klassischen CT nicht oder nur schwierig zu beurteilen ist.

\section{Reduktion von Metallartefakten}

Das Ausmaß der Artefakte ist von der Metalllegierung abhängig. Titan verursacht weniger Artefakte als Kobalt-Chrom. Am meisten Artefakte kommen bei „Stainless-steel“-Komponenten vor [5]. Dies ist bedingt durch die unterschiedlichen Massenschwächungskoeffizienten. Wenn möglich, soll die Längsachse eines Metallimplantats in Tischrichtung positioniert werden, was nicht immer möglich ist.

Akquisitionsparameter. Höhere kV-Werte erhöhen die Wahrscheinlichkeit, dass Röntgenstrahlen die Prothese passieren, was die Artefakte nur leicht vermindert [6,7], jedoch zu einer Dosiserhöhung führt. Erhöht man die mAs, erreichen mehr Photonen den Detektor (siehe „photon starvation“). Dies bedeutet aber ebenfalls eine höhere Strahlendosis für den Patienten. Für die Metallartefaktreduktion ist der Effekt ebenfalls eher gering [7]. Durch eine enge Kollimation können die Artefakte ebenfalls reduziert werden, v. a. durch weniger Partialvolumeneffekte [1].

Rekonstruktionsparameter und -algorithmen. Wie oben erwähnt, ist bei der Bildakquisition eine dünne Kollimation vorteilhaft. Für die sekundäre Rekonstruktion hingegen sind dickere Schichten besser und können insbesondere das Bildrauschen reduzieren [1]. Die Erweiterung der oberen Begrenzung der Hounsfield-Skala von 4000 auf 40.000 („extended CT scale“) kann die Bildqualität bei Metallartefakten ebenfalls etwas verbessern [8]. Iterative Rekonstruktionen werden in den letzten Jahren vermehrt eingesetzt [9]. Hierbei wird bei der Rekonstruktion der CT-Bilder aus den Rohdaten eine Korrekturschleife eingeführt, die mehrfach durchlaufen wird und so Bildrauschen und Artefakte reduzieren kann. Hilfreich ist das z. B. für Weichteile im Becken zwischen 2 Hüftprothesen [10]. Die Beurteilung des Metall-Knochen-Übergangs ist weiterhin ein Problem, da durch die Software z. T. Details verloren gehen [11]. Prinzipiell funktionieren solche Rechenalgorithmen umso besser, je größer das Artefakt ist [12]. Der Algorithmus lässt sich auch mit Dual-Energy verbinden [13].

Das Ausmaß der Artefakte ist von der Metalllegierung abhängig 
Dual-Energy. Dual-Energy-CT kann erfolgreich zur Metallartefaktreduktion eingesetzt werden [14]. Bei einem normalen CT ist der Röntgenstrahl, wie oben erwähnt, polychromatisch (d. h. mit einem breiten Energiespektrum). Der Energiedurchschnitt bei monochromatischen Röntgenstrahlen (d. h. alle mit dem gleichen keV-Wert) bleibt gleich und ist nicht durch Aufhärtung beeinflusst. Dual-Energy nimmt 2 Datensets derselben Körperregion mit unterschiedlichen kV-Peaks auf [normalerweise 80 (oder 100) kVp und $140 \mathrm{kVp}$ ]. Daraus lassen sich beliebige monochromatische Bilder errechnen [15]. Tiefere Energielevels um die $80 \mathrm{keV}$ sind gut für die Beurteilung der Weichteile, während höhere Werte (130-140 keV) für Metall und Knochen vorteilhaft sind [15].

\section{Magnetresonanztomographie}

\section{Metallinduzierte Artefakte}

Für eine gute Bildqualität ist ein homogenes Magnetfeld wichtig. Die Präsenz von Metall im Magnetfeld führt zu Verzerrungen und zu entsprechenden Artefakten [16]. Das Signal kann durch diese Verzerrungen beim Auslesen am falschen Ort erscheinen (Signalverschiebung), zudem tritt ein Signalverlust ( „signal loss“) oder eine lokale Signalvermehrung ( „pile-up“) auf [17]. Verzerrungen können jedoch nicht nur innerhalb der Bildebene auftreten ( „in-plane distortion“), sondern auch aus benachbarten Schichten tauchen Artefakte in der Bildebene auf ( „through-plane distortion“).

Des Weiteren ist die Fettsättigung erschwert, was insbesondere die spektrale Fettsättigung betrifft. Die STIR-Technik ist etwas weniger anfällig [17].

\section{Reduktion von Metallartefakten}

Die Gerätewahl kann mithelfen die Metallartefakte zu reduzieren. So sind die Artefakte bei 1,5 T deutlich geringer als bei $3 \mathrm{~T}$ [18].

Durch den Einsatz von Fast-Spin-Echo-Sequenzen kann man Signalverlust durch Dephasierung gegenüber anderen Sequenzen reduzieren. Für eine bessere Fettsättigung empfiehlt sich die STIR-Sequenz oder die Dixon-Technik. V. a. STIR führt in Metallnähe zu einer homogeneren Fettsättigung, allerdings bei einer tieferen SNR („signal-to-noise ratio“; [17]).

Die geometrische und „In-plane“-Distortion kann mit einer höheren Empfängerbandbreite reduziert werden [17]. Eine weitere Art, um „In-plane“-Artefakte zu korrigieren, ist das sog. „viewangle tilting" (VAT), bei welchem ein zusätzlicher Kompensationsgradient während der Auslesephase angewendet wird. Dies führt zu einer kompensatorischen Verlagerung der Schichtauswahlebene, um die durch das Metall verursachten Verzerrungen innerhalb der Bildebene zu korrigieren [19]. VAT kann allerdings zu einer vermehrten Bildunschärfe führen [20]. Dünnere Schichten können helfen, die „through-plane distortion“ zu reduzieren, was aber längere Untersuchungszeiten und reduzierte SNR bedeutet.

Neuere Methoden, welche neben „In-plane“-Artefakten auch „Through-plane“-Artefakte reduzieren, sind MAVRIC („multi-acquisition variable-resonance image combination“; [21]) und SEMAC („slice-encoding for metal artifact correction“; - Abb. 1, [22]). Bei MAVRIC werden mehrere Schichtstapel in unterschiedlichen Frequenzen akquiriert und am Ende die Signale der verzerrten Spins für die Artefaktreduktion kombiniert. Bei SEMAC werden diese Schichtstapel mit einer Frequenz, jedoch mit einer zusätzlichen Kodierung in Schichtwahlrichtung akquiriert, um die Verzerrung der angrenzenden Bilder zu reduzieren. Sutter et al. [23, 24] konnten zeigen, dass SEMAC im klinischen Einsatz gut funktioniert. In der präklinischen Entwicklung sind zudem weitere Methoden wie z. B. ultraschnelle TE-Sequenzen [25] oder SWIFT („sweep imaging with Fourier transformation“; [26]). Eine praktische Übersicht mit Möglichkeiten der Metallartefaktreduktion zeigt • Tab. 1. 


\begin{tabular}{|lll}
\hline Tab. 2 & Grading nach & Anderson [46] für Patienten mit Metall-Metall-Prothesen \\
\hline Grad & Beschreibung & Kriterien \\
\hline A & $\begin{array}{l}\text { Normal oder } \\
\text { Akzeptabel }\end{array}$ & Normale postoperative Veränderungen (Serome und kleine Hämatome) \\
\hline B & Infektion & $\begin{array}{l}\text { Flüssigkeitskollektion mit T2-hyperintensem Randwall, entzündliche } \\
\text { Weichteilveränderungen } \pm \text { Knochenmarködem }\end{array}$ \\
\hline C1 & Gering & Periprothetische Weichteilmasse oder Flüssigkeitskollektion mit Durchmesser $<5 \mathrm{~cm}$ \\
\hline C2 & Moderat & $\begin{array}{l}\text { Periprothetische Weichteilmasse oder Flüssigkeitskollektion mit Durchmesser }>5 \mathrm{~cm} \\
\text { oder } \\
\text { C1-Läsion mit entweder Muskelatrophie, Muskelödem oder Knochenmarködem }\end{array}$ \\
\hline C3 & Schwer & $\begin{array}{l}\text { Mindestens einer der folgenden Punkte: } \\
\text { - flüssigkeitsgefüllte Höhle mit Durchbruch durch die tiefen Faszien } \\
\text { - Sehnenavulsion } \\
\text { - Fraktur }\end{array}$ \\
\hline
\end{tabular}

Tab. 3 Brooker-Klassifikationssystem für

heterotope Ossifikationen [58]

Grad I Knocheninseln im Weichteilgewebe um das Hüftgelenk

Grad II Knochenappositionen vom Becken oder proximalen Femur mit $\geq 1 \mathrm{~cm}$ Abstand zwischen Becken und Femur

Grad III Knochenappositionen vom Becken oder proximalen Femur mit $<1 \mathrm{~cm}$ Abstand zwischen Becken und Femur

Grad IV Ankylosierende Ossifikationen um das Hüftgelenk

\section{Pathologien}

\section{Lockerung}

Eine Lockerung der Hüftprothese ist der häufigste Grund für eine Revision [27]. Ursachen können neben dem Verlust der mechanischen Fixierung auch partikelinduzierte Osteolysen sein (siehe unten). Bei Verdacht auf Lockerung wird primär ein Röntgenbild angefertigt. Feine Lysesäume bis $2 \mathrm{~mm}$, die im Verlauf stationär bleiben, gelten noch als normal. Über $2 \mathrm{~mm}$ spricht man von möglicher Lockerung. Ist der Lysesaum progredient oder ändern die Metallkomponenten ihre Position, handelt es sich um eine definitive Lockerung. Bei nichtzementierten Prothesen kann ein geringes Einsinken von weniger als $10 \mathrm{~mm}$ der Femurkomponente in den ersten Monaten noch normal sein [28].

Die Lysesäume sind je nach Größe häufig mit der CT zu erkennen. Die MRT ist für das Erkennen von schmalen Lysesäumen weniger geeignet, da der unmittelbare Prothesen-Knochen(-Zement)Übergang aufgrund von Metallartefakten auch mit aktuellen metallartefaktreduzierenden Sequenzen nur eingeschränkt zu beurteilen ist.

\section{Abriebpartikel}

Frühestes Zeichen in der MRT bei Abriebpartikeln ist eine partikelinduzierte Synovitis mit Gelenkerguss, was jedoch nicht zwingend zu Symptomen führt [29]. Die Synovia ist verdickt, und hypointenser Debris ist innerhalb des Gelenks zu sehen.

\section{Periprothetische Osteolysen}

Bei Metall-Polyethylen-Prothesen gelangen durch Abnutzung und Abrieb am Polyethylen-Inlay winzige Partikel ins Gelenk respektive zwischen Prothese und Knochen. Diverse Zellen wie z. B. Makrophagen werden dadurch aktiviert. Diese initiieren eine biologische Reaktion, welche zu periprothetischen Osteolysen führt [30]. Je nach Größe der Osteolysen verliert die Hüftprothese ihre mechanische Stabilität.

Die CT ist in dieser Hinsicht dem konventionellen Röntgenbild überlegen [31, 32]. In der CT sind die Osteolysen als gut abgrenzbare Areale ohne Knochenstruktur zu erkennen. Typischerweise haben die Osteolysen Verbindung respektive Kontakt zum Prothesenmaterial. Die Dichtewerte liegen um die $30 \mathrm{HU}$ (-40 bis $100 \mathrm{HU}$ ) und zeigen nach Kontrastmittelgabe Dichtewerte um die $90 \mathrm{HU}$ [33].

In der MRT sind die Osteolysen T1-hypointens, mit intermediärem bis hyperintensem Signal in T2 und hypointensem Randsaum zum Knochen hin (• Abb. 2; [33]). Nach Kontrastmittelgabe zei-
Eine Lockerung der Hüftprothese ist der häufigste Grund für eine Revision

Die MRT ist für das Erkennen von schmalen Lysesäumen weniger geeignet

Frühestes Zeichen in der MRT bei Abriebpartikeln ist eine partikelinduzierte Synovitis mit Gelenkerguss 

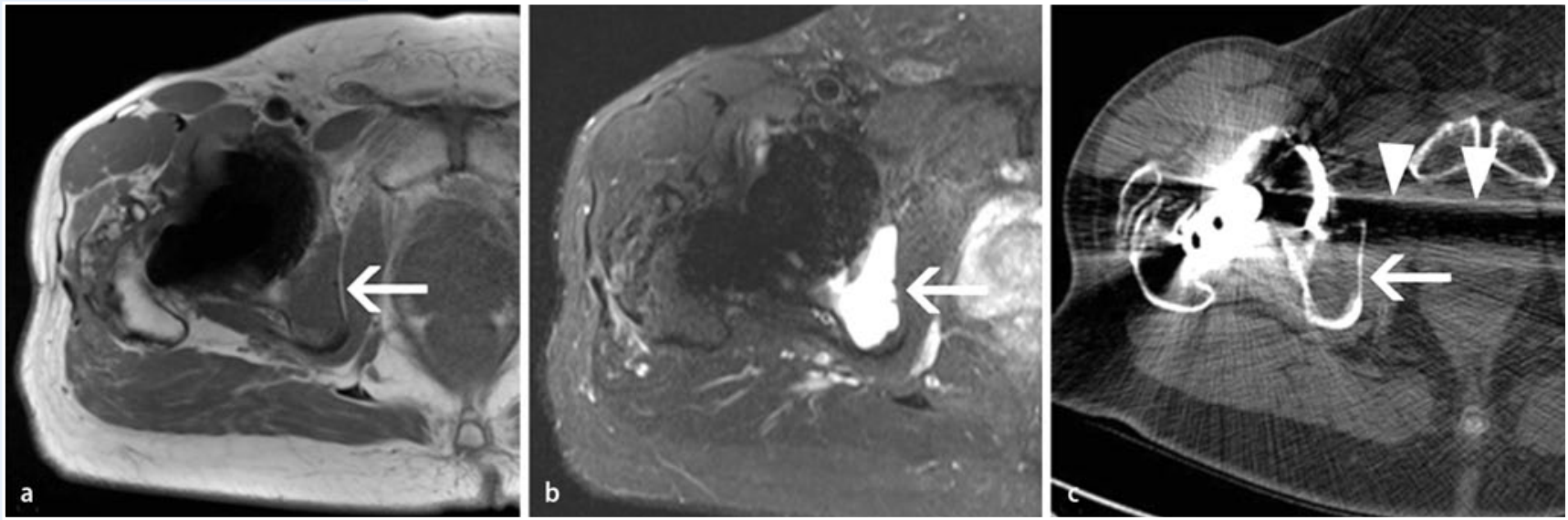

Abb. $2 \Delta$ Magnetresonanztomographie und Computertomographie (CT) des rechten Hüftgelenks in transversaler Ebene: Große Osteolyse (weißer Pfeil) im Acetabulum rechts bei Hüftprothese, in T1-Sequenz (a) hypointens, in STIR-Sequenz (b) deutlich hyperintens, in der CT (c) keine Trabekel sichtbar, aber aufgrund der Aufhärtungsartefakte (Pfeilspitzen) schwierig zu sehen
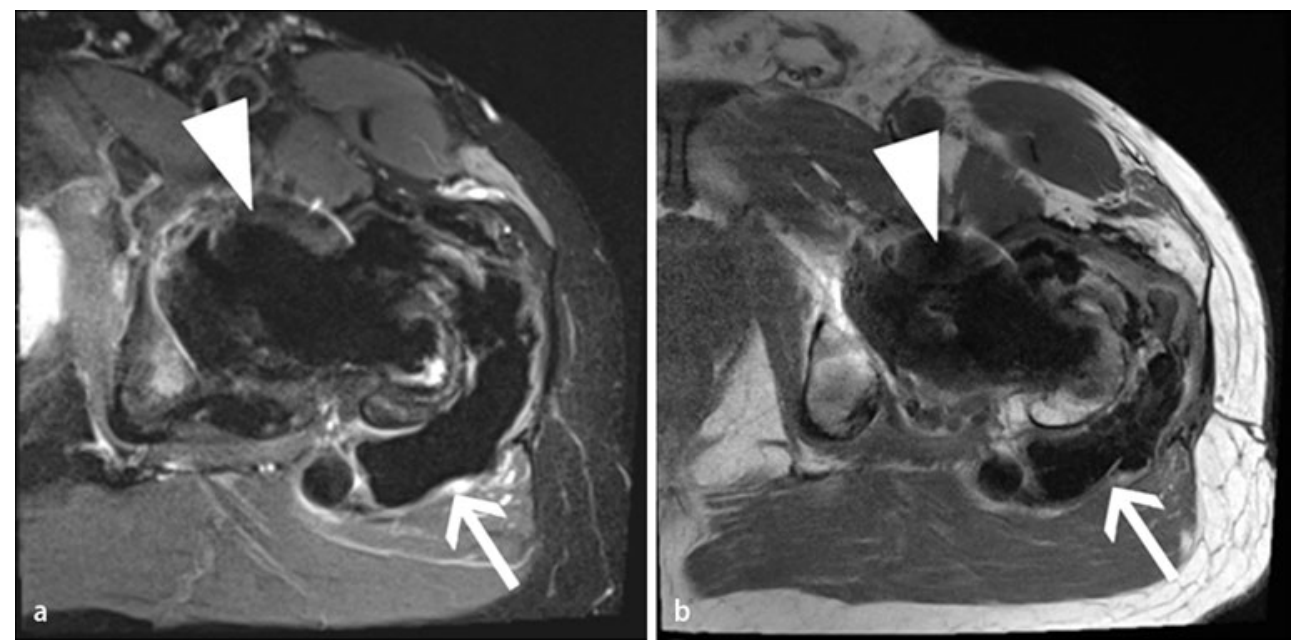

Abb. $3 \Delta$ Magnetresonanztomographie des linken Hüftgelenks in transversaler Ebene bei einem Patienten mit Hüftprothese und Metallose: a STIR-Sequenz, b T1-gewichtete Sequenz. In beiden Sequenzen stark hypointenses Material um das linke Hüftgelenk (weißer Pfeil), Artefakt durch Hüftprothese (weiße Pfeilspitze)

Die MRT ist der CT und dem

Röntgenbild bei der Detektion von Osteolysen im Becken überlegen gen die Osteolysen peripheres und z. T. auch ein wenig zentrales Enhancement [34]. Die MRT ist der CT und dem Röntgenbild bei der Detektion von Osteolysen im Becken überlegen [35].

\section{Metallose}

Die Metallose ist eine Komplikation nach Gelenkersatz, bei dem es durch Metallabrieb zu Ablagerungen von Metallpartikeln ins umliegende Gewebe kommt. Ursächlich sind unter anderem vollständiger Abrieb, abnormer Kontakt z. B. zwischen Acetabulumrand und Prothesenhals, Dislokation des Polyethylen-Inlays oder die Verwendung von Metall-Metall-Prothesen. Die Metallose wird diagnostiziert durch Nachweis von Metallabriebpartikeln bei Gelenkpunktion (schwarze Flüssigkeit) sowie Nachweis von dunklen Gewebeveränderungen im Rahmen einer Revisionsoperation oder bei der histologischen Aufarbeitung [36].

Das Röntgenbild kann unauffällig sein. In der CT kann man gelegentlich dichtes Abriebmaterial erkennen. In der MRT können periprothetische Ansammlungen von stark hypointensem Material oder Flüssigkeitskollektionen mit stark hypointensem Randwall auf eine Metallose hinweisen (• Abb. 3; [37]). 

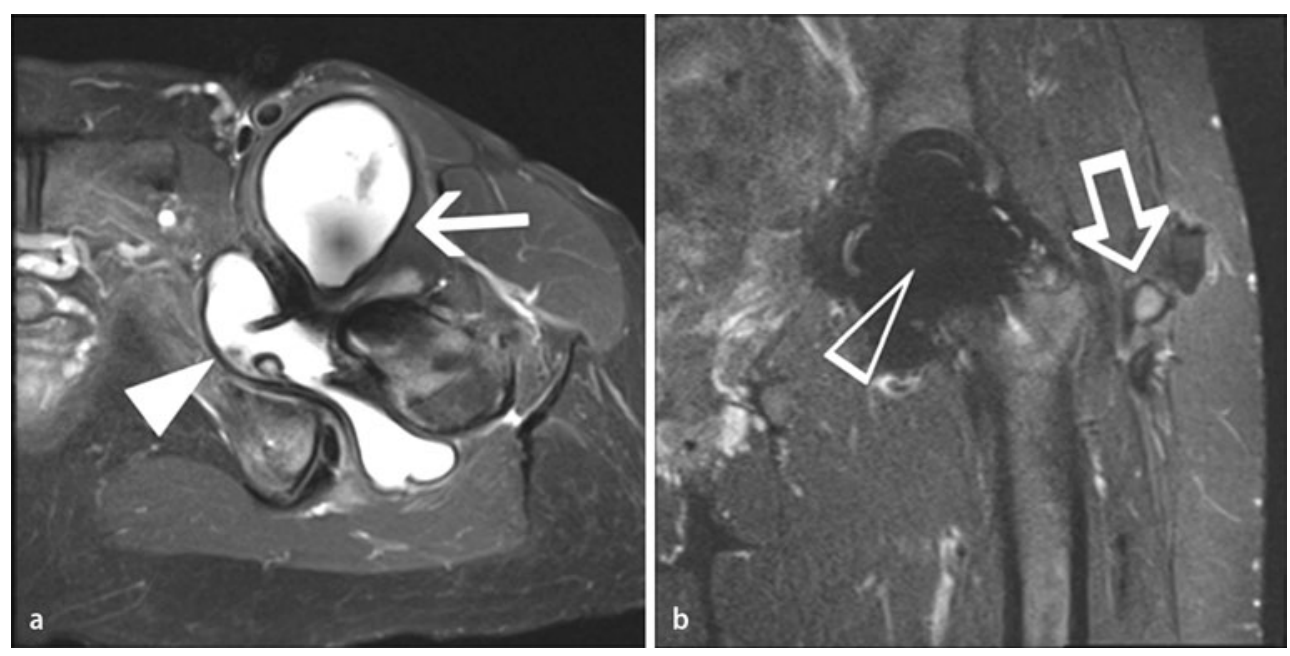

Abb. $4 \Delta$ Magnetresonanztomographie mit STIR-Sequenzen zeigen Pseudotumoren bei 2 unterschiedlichen Patienten mit Oberflächenersatz am linken Hüftgelenk: $\mathbf{a}$, weißer Pfeil große komplexe Flüssigkeitskollektion (= Pseudotumor) mit stark hypointensem Randwall (weiße Pfeilspitze); b kleiner Pseudotumor lateral des Trochanter major (offener weißer Pfeil), Metallartefakt durch Oberflächenersatz (offene weiße Pfeilspitze)

\section{Metall-Metall-Problematik/Pseudotumoren}

Die weit verbreiteten und seit den 1960er-Jahren eingesetzten Systeme mit acetabulärem Polyethylen-Inlay haben gute Langzeitresultate gezeigt: 80\% dieser Implantate halten länger als 25 Jahre. Bei jüngeren Patienten ist die Haltbarkeit aber reduziert. Man geht davon aus, dass dies durch vermehrte körperliche Aktivität bedingt ist [38]. Um dieser vermehrten Abnützung entgegenzutreten, wurden neuere System mit härterem Material eingeführt wie Keramikkopf in Keramik, Metallkopf und Metallpfanne („metal-on-metal“) oder hochvernetztes Polyethylen. .Im Vergleich zu Metall-Polyethylen zeigten Metall-Metall-Prothesen in vitro weniger Abnützung [39]. So wurde im letzten Jahrzehnt eine beträchtliche Anzahl Metall-Metall-Prothesen eingebaut. Die Abriebpartikel sind im Vergleich zum Polyethylen deutlich kleiner und zahlreicher. Diese Metallionen gelangen ins Gelenk und in den Blutkreislauf [40]. 2005 beschrieben Willert et al. [41] erstmals vermehrte perivaskuläre Lymphozytenund Plasmazellinfiltrate um Metall-Metall-Prothesen. 2008 wurden bei Patienten mit Metall-MetallProthesen Weichteilmassen in Prothesennähe gefunden und als Pseudotumoren benannt, histologisch aus Nekrosen und Lymphozyteninfiltraten bestehend [42]. Als mögliche Ursachen werden Reaktionen auf Abriebpartikel oder eine Metallhypersensitivität diskutiert [43]. Ab 2010 haben mehrere Hersteller und Behörden Warnungen herausgegeben und einzelne Systeme vom Markt nehmen müssen. Pseudotumoren bei Metall-Metall-Prothesen sind häufig (bis 69\%) und interessanterweise nicht unbedingt mit Schmerzen assoziiert [44]. Assoziiert mit Schmerzen sind eher Knochenmarködeme und Sehnenrisse [44]. Es wurden mehrere Grading-Systeme von Weichteilveränderungen bei Metall-Metall-Prothesen vorgeschlagen, wobei das Klassifikationssystem nach Anderson (• Tab. 2) als das zuverlässigste gilt [45].

In der MRT präsentieren sich Pseudotumoren als periprothetische Flüssigkeitskollektionen jeglicher Größe. Sie können flüssigkeitsäquivalentes Signal aufweisen oder als solide Masse erscheinen (• Abb. 4; [44]). Pseudotumoren können einen stark hypointensen Randwall aufweisen.

\section{Infektionen}

Ein weiterer häufiger Grund für Revisionsoperationen bei Hüftprothesen sind Infektionen [27, 47]. Die klinische Diagnose eines Protheseninfekts kann schwierig sein. Die wichtigste diagnostische Maßnahme ist die Gelenkpunktion mit Zellzahlbestimmung. Konventionelle Röntgenbilder sind oft normal. In der CT kann eine aggressive Osteolyse mit unscharfen Rändern (im Sinne einer Osteomyelitis) auf einen Infekt hinweisen. Gemäß Cyteval et al. [48] ist ein wichtiger Hinweis auf einen Infekt eine Periostitis. Periprothetische Knochenveränderungen helfen bei der Unterscheidung zwi-
Im Vergleich zu Metall-Polyethylen zeigten Metall-Metall-Prothesen in vitro weniger Abnützung

Pseudotumoren bei MetallMetall-Prothesen sind häufig

Ein häufiger Grund für Revisionsoperationen bei Hüftprothesen sind Infektionen 

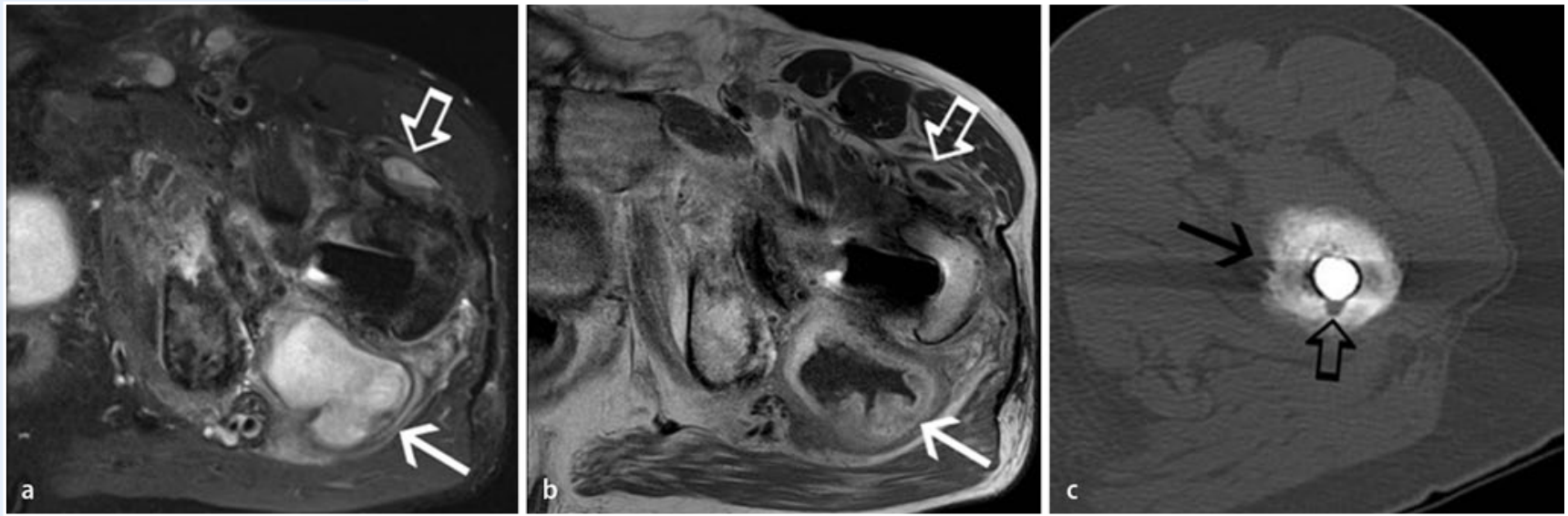

Abb. $5 \Delta$ Magnetresonanztomographie und Computertomographie in transversaler Ebene bei Hüftinfekt bei 2 unterschiedlichen Patienten (a STIR-Sequenz, b T1-gewichtete Sequenz nach Kontrastmittelgabe): großer Abszess mit deutlich kontrastmittelaufnehmender dicker Kapsel (weißer Pfeil), ein kleinerer Verhalt ventral des Trochanter major (offener weißer Pfeil). c Zweiter Patient mit Protheseninfekt: deutliche Periostreaktion (schwarzer Pfeil) sowie kleine Lysezone am Prothesenschaft (offener schwarzer Pfeil)
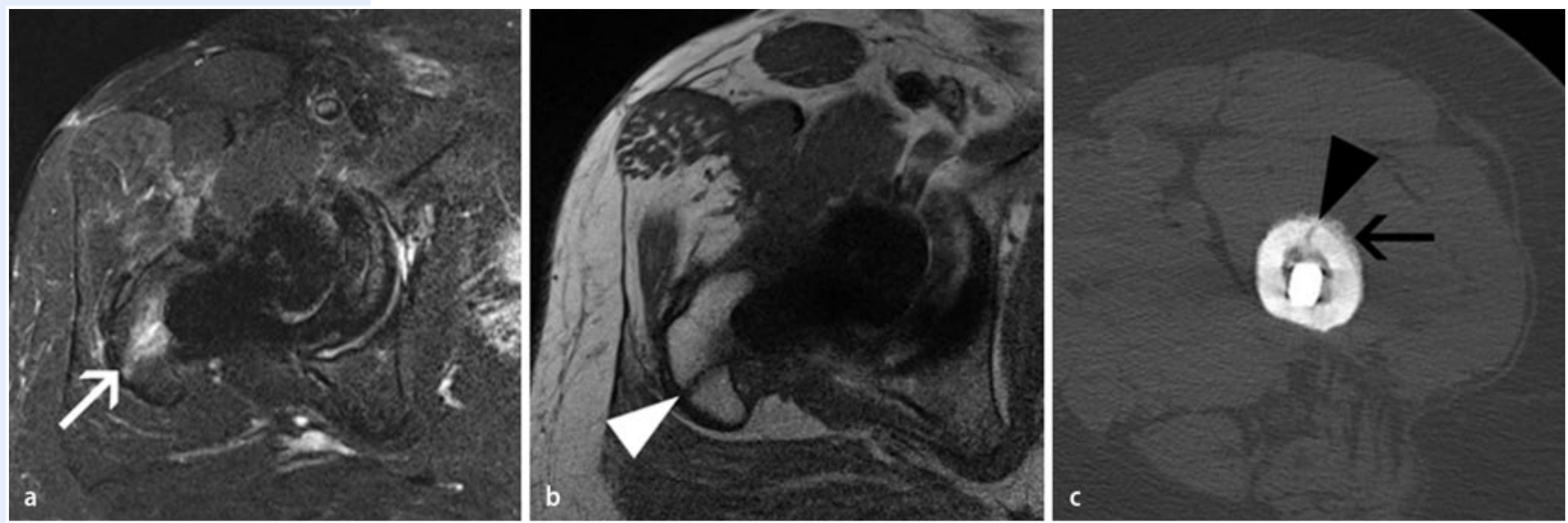

Abb. $6 \Delta$ Magnetresonanztomographie $(\mathbf{a}, \mathbf{b})$ des rechten Hüftgelenks in transversaler Ebene und Computertomographie (c) des linken Femurs proximal auf Höhe des Prothesenschafts bei 2 Patienten mit periprothetischen Frakturen: a STIR-Sequenz mit Knochenmarködem im Frakturbereich (weißer Pfeil), b T1-gewichtete Sequenz mit hypointenser Frakturlinie (weiße Pfeilspitze), c anderer Patient mit Fissur am Femurschaft (schwarze Pfeilspitze) und lokaler Periostreaktion (schwarzer Pfeil)

schen Infekt oder Lysezone wenig. Eine rasche morphologische Veränderung einer Osteolyse weist auf einen Infekt hin. Die CT ist z. T. hilfreich bei der Beurteilung der Weichteile um die Hüftprothese. Insbesondere größere Flüssigkeitskollektionen in Muskeln und im umgebenden Fettgewebe können auf einen Infekt hinweisen [48].

Die MRT kann die Ausbreitung des Infekts in die umliegenden Weichteile gut darstellen (• Abb. 5; [49]). Bei einem Infekt kann die Synovia hyperintens sein und einen lamellierten Aspekt aufweisen [50]. Im Unterschied zu Pseudotumoren zeigen Infektkollektionen keinen stark hypointensen Randwall.

\section{Frakturen}

Periprothetische Frakturen sind häufiger bei nichtzementierten Prothesen
Periprothetische Frakturen sind häufiger bei nichtzementierten Prothesen [51]. Ist das konventionelle Röntgenbild unklar, hilft die CT weiter. Die MRT hat ihre Stärke bei okkulten Insuffizienzfrakturen an Schambeinästen oder Sakrum. Bei unklaren Schmerzen ist eine flüssigkeitssensitive Sequenz (z. B. STIR) mit großem "field of view“ im Beckenbereich hilfreich. Mit metallartefaktreduzierenden Sequenzen (z. B. SEMAC) lassen sich in der MRT auch periprothetische Frakturen diagnostizieren (• Abb. 6). 


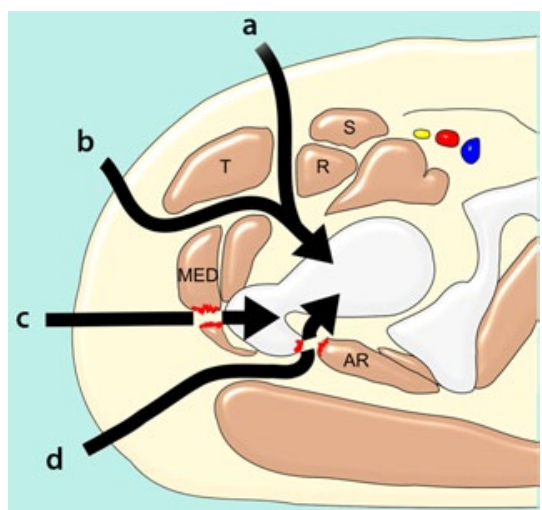

Abb. $7 \triangle$ Graphische Darstellung der operativen Zugangswege bei Hüftgelenksersatz [ $a$ anteriorer Zugang, $b$ anterolateraler Zugang, $c$ transglutealer (lateraler) Zugang, $d$ posteriorer Zugang, $T \mathrm{M}$. tensor fasciae latae, $R \mathrm{M}$. rectus femoris, $S$ M. Sartorius, MED M. gluteus medius, AR Außenrotatoren (M. obturatorius internus, M. piriformis]

wichteten Sequenzen gut sichtbar sind. Die Muskelqualität sollte bei allen Patienten mitbeurteilt werden. Verfettungen des M. gluteus minimus sind nach lateralem Zugang häufig, auch bei asymptomatischen Patienten. Verfettungen v. a. des posterioren Anteils des M. gluteus medius kommen hingegen praktisch nur bei symptomatischen Patienten vor [53].

\section{Anteriores Iliopsoassehnen-Impingement nach Hüftprothese}

Die Sehne des M. iliopsoas kann durch rezidivierendes Scheuern am Vorderrand der Prothesenpfanne irritiert werden, wenn das Metall über den anterioren ossären Acetabulumrand hinausragt (• Abb. 8; [54]). Die fehlende ossäre Deckung und der Überstand der Pfanne können in der CT abgebildet werden. In der MRT kann unter Umständen eine Reizung der Iliopsoassehne erkannt werden.

\section{Heterotope Ossifikationen}

Heterotope Ossifikationen entstehen in einer Häufigkeit von bis zu $43 \%$ nach Hüftprothesenoperationen [55] und können schmerzhaft sein. Mögliche Behandlungen sind nichtsteroidale entzündungshemmende Schmerzmittel (Indometacin; [56]) oder prophylaktische Bestrahlung [57]. Die Verkalkungen sind im konventionellen Röntgenbild und in der CT einfach nachzuweisen und werden nach Brooker klassifiziert (• Tab. 3; [58]).

Die MRT kann hilfreich sein, um die genaue Ausdehnung und die Beziehung der heterotopen Ossifikation zu umgebenden Strukturen wie Gefäßen und Nerven zu beschreiben. Es wird zwischen unreifen und reifen heterotopen Ossifikationen unterschieden. Unreife Anteile zeigen noch kein Fettsignal, können liquide aussehen und sollten nicht mit einer Infektion oder einem Tumor verwechselt werden. Heterotope Ossifikationen können v. a. initial ein starkes ringförmiges Kontrastmittel-Enhancement zeigen. Mit zunehmender Ausreifung sinkt das T2-Signal ab, und die heterotopen Ossifikationen nehmen immer weniger Kontrastmittel auf. Zeitgleich entstehen immer mehr Areale, welche sich vom Signal her wie normaler Knochen verhalten [59].

\section{Nervenschädigungen}

Durch die Operation (Schnittverletzung, Hämatome), durch implantiertes Material wie Schrauben im Acetabulum sowie durch Narbengewebe und heterotope Ossifikationen können Nerven um das Hüftgelenk gereizt oder geschädigt werden. Die MRT ist hier die beste Methode, um Auffälligkeiten im Verlauf der Nerven oder, im Falle einer Denervation, im Muskel darzustellen. Auch an die Möglichkeit eines Neuroms sollte gedacht werden.
Eine wichtige Information ist der verwendete operative Zugangsweg

Die Muskelqualität sollte bei allen Patienten mitbeurteilt werden

Es wird zwischen unreifen und reifen heterotopen Ossifikationen unterschieden

Die MRT ist die beste Methode, um Auffälligkeiten im Verlauf der Nerven oder im Muskel darzustellen 


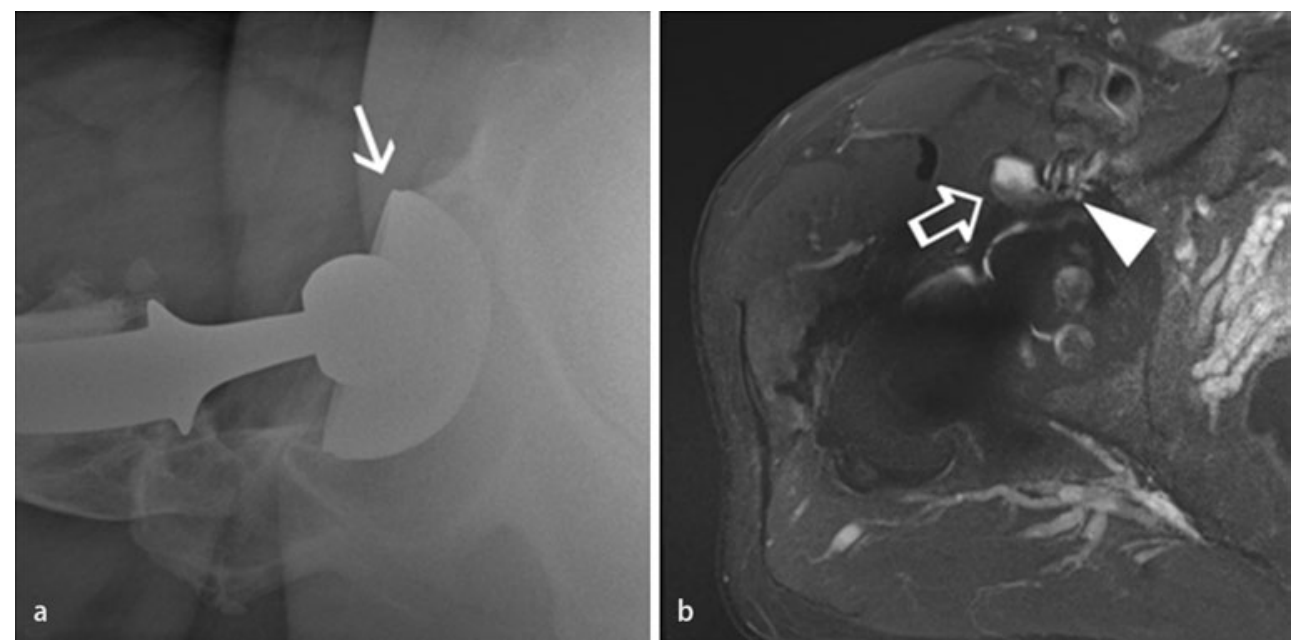

Abb. $8 \Delta$ a Axiales Röntgenbild bei Patient mit Hüftprothese rechts: verminderte Anteversion der Acetabulumkomponente mit hervorstehendem anterioren Metallrand (weißer Pfeil); $\boldsymbol{b}$ lliopsoassehnen-Impingement mit Erguss in der Bursa iliopectinea (offener, weißer Pfeil) und streifiger Reizung der lliopsoassehne (weiße Pfeilspitze)

\section{Zusammenfassung}

Durch die technischen Fortschritte in der Metallartefaktreduktion für CT und MRT kann man beide Verfahren bei Patienten mit Hüftprothese heute mit guten Resultaten einsetzen. Beide Methoden helfen bei der Abklärung bei Patienten mit schmerzhaften Hüftprothesen. Partikelinduzierte Osteolysen können zu einer Lockerung der Hüftprothese führen. Bei Metall-Metall-Prothesen sollte man auf die Bildung von Pseudotumoren achten. Infektionen, Frakturen und Weichteilveränderungen sind in die Differenzialdiagnose bei schmerzhafter Hüftprothese einzubeziehen.

Bei Frage nach Vorstehen der acetabulären Komponente sowie bei Frage nach Durchbau, Frakturen und Fissuren hilft primär die CT weiter. Bei Frage nach ossären Stressreaktionen oder Weichteilveränderungen (Sehnen, Muskeln) ist die MRT vorteilhaft. Für die Abklärung von Osteolysen können je nach Möglichkeiten der Metallartefaktreduktion die CT oder die MRT eingesetzt werden.

\section{Fazit für die Praxis}

- Eine Hüftprothese ist keine Kontraindikation für eine MRT der Hüfte.

- Mit entsprechender Metallartefaktreduktion können die CT und die MRT erfolgreich zur Abklärung von schmerzhaften Hüftprothesen eingesetzt werden. In der CT können insbesondere iterative Rekonstruktionsalgorithmen oder Dual-Energy-CT und in der MRT neuere metallartefaktreduzierende Sequenzen (SEMAC oder MAVRIC) helfen, die Artefakte zu minimieren.

- Osteolysen durch Polyethylenabrieb können zu einer Lockerung der Hüftprothese führen. Diese Osteolysen können zuverlässig mit CT und MRT bei Einsatz von metallartefaktreduzierender Technik diagnostiziert werden.

- Bei Metall-Metall-Prothesen können durch Metallabrieb sog. Pseudotumoren entstehen. Diese sind aber oft asymptomatisch. Schmerzen sind eher mit gleichzeitig vorhandenen Knochenmarködemen oder Sehnenschäden assoziiert.

\section{Korrespondenzadresse}

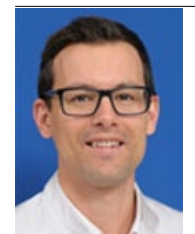

Dr. C.A. Agten

Radiologie, Uniklinik Balgrist,

Forchstr. 340, CH-8008 Zürich

christoph.agten@balgrist.ch 


\section{Einhaltung ethischer Richtlinien}

Interessenkonflikt. CA. Agten, R. Sutter und C.W.A. Pfirrmann geben an, dass kein Interessenkonflikt besteht.

Dieser Beitrag beinhaltet keine Studien an Menschen oder Tieren.

\section{Literatur}

1. Barrett JF, Keat N (2004) Artifacts in CT: recognition and avoidance. Radiographics 24:1679-1691

2. Boas FE, Fleischmann D (2012) CT artifacts: causes and reduction techniques. Imaging Med 4:229-240

3. Mori I, Machida Y, Osanai M et al (2013) Photon starvation artifacts of $\mathrm{X}$-ray $\mathrm{CT}$ : their true cause and a solution. Radiol Phys Technol 6:130-141

4. Joseph PM, Spital RD (1982) The effects of scatter in x-ray computed tomography. Med Phys 9:464-472

5. Haramati N, Staron RB, Mazel-Sperling Ket al (1994) CT scans through metal scanning technique versus hardware composition. Comput Med Imaging Graph 18:429-434

6. Lee M-J, Kim S, Lee SA et al (2007) Overcoming artifacts from metallic orthopedic implants at high-field-strength MR imaging and multi-detector $C T$. Radiographics 27:791-803

7. Moon SG, Hong SH, Choi JY et al (2008) Metal artifact reduction by the alteration of technical factors in multidetector computed tomography: a 3-dimensional quantitative assessment. J Comput Assist Tomogr 32:630-633

8. Link TM, Berning W, Scherf S et al (2000) CT of metal implants: reduction of artifacts using an extended CT scale technique. J Comput Assist Tomogr 24:165-172

9. Boas FE, Fleischmann D (2011) Evaluation of two iterative techniques for reducing metal artifacts in computed tomography. Radiology 259:894-902

10. Morsbach F, Bickelhaupt $S$, Wanner GA et al (2013) Reduction of metal artifacts from hip prostheses on $\mathrm{CT}$ images of the pelvis: value of iterative reconstructions. Radiology 268:237-244

11. Malan DF, Botha CP, Kraaij G et al (2012) Measuring femoral lesions despite CT metal artefacts: a cadaveric study. Skeletal Radiol 41:547-555

12. Liu P, Pavlicek W, Peter M et al (2009) Metal artifact reduction image reconstruction algorithm for $\mathrm{CT}$ of implanted metal orthopedic devices: a work in progress. Skeletal Radiol 38:797-802

13. Lee YH, Park KK, Song HT et al (2012) Metal artefact reduction in gemstone spectral imaging dual-energy $\mathrm{CT}$ with and without metal artefact reduction software Eur Radiol 22:1331-1340

14. Lewis M, Reid K, Toms AP (2013) Reducing the effects of metal artefact using high keV monoenergetic reconstruction of dual energy CT (DECT) in hip replacements. Skeletal Radiol 42:275-282

15. Pessis E, Campagna R, Sverzut JM et al (2013) Virtual monochromatic spectral imaging with fast kilovoltage switching: reduction of metal artifacts at CT. Radiographics 33:573-583
16. Schenck JF (1996) The role of magnetic susceptibility in magnetic resonance imaging: MRI magnetic compatibility of the first and second kinds. Med Phys 23:815-850

17. Hargreaves $B A$, Worters PW, Pauly KB et al (2011) Metal-induced artifacts in MRI. AJR Am J Roentgenol 197:547555

18. Graf H, Lauer UA, Berger A et al (2005) $\mathrm{RF}$ artifacts caused by metallic implants or instruments which get more prominent at $3 \mathrm{~T}$ : an in vitro study. Magn Reson Imaging 23:493-499

19. Cho ZH, Kim DJ, Kim YK (1988) Total inhomogeneity correction including chemical shifts and susceptibility by view angle tilting. Med Phys 15:7-11

20. Butts K, Pauly JM, Gold GE (2005) Reduction of blurring in view angle tilting MRI. Magn Reson Med 53:418-424

21. Koch KM, Lorbiecki JE, Hinks RS et al (2009) A multispectral three-dimensional acquisition technique for imaging near metal implants. Magn Reson Med 61:381-390

22. Lu W, Pauly KB, Gold GE et al (2009) SEMAC: slice encoding for metal artifact correction in MRI. Magn Reson Med 62:66-76

23. Sutter R, Hodek R, Fucentese SF et al (2013) Total knee arthroplasty MRI featuring slice-encoding for metal artifact correction: reduction of artifacts for STIR and proton density-weighted sequences. AJR Am J Roentgenol 201:1315-1324

24. Sutter R, Ulbrich EJ, Jellus V et al (2012) Reduction of metal artifacts in patients with total hip arthroplasty with sliceencoding metal artifact correction and view-angle tilting MR imaging. Radiology 265:204-214

25. Gold GE, Thedens DR, Pauly JM et al (1998) MR imaging of articular cartilage of the knee: new methods using ultrashort TEs. AJR Am J Roentgenol 170:1223-1226

26. Idiyatullin D, Corum C, Park JY et a (2006) Fast and quiet MRI using a swept radiofrequency. J Magn Reson 181:342-349

27. Melvin JS, Karthikeyan T, Cope R, Fehring TK (2013) Early failures in total hip arthroplasty - a changing paradigm. J Arthroplasty 29:1285-1288

28. Manaster BJ (1996) From the RSNA refresher courses. Total hip arthroplasty: radiographic evaluation. Radiographics 16:645-660

29. Cooper HJ, Ranawat AS, Potter HG et al (2010) Early reactive synovitis and osteolysis after total hip arthroplasty. Clin Orthop Relat Res 468:3278-3285

30. Gallo J, Goodman SB, Konttinen YT et al (2013) Particle disease: biologic mechanisms of periprosthetic osteolysis in total hip arthroplasty. Innate Immun 19:213-224
31. Leung S, Naudie D, Kitamura $\mathrm{N}$ et al (2005) Computed tomography in the assessment of periacetabular osteolysis. J Bone Joint Surg Am 87:592-597

32. Puri L, Wixson RL, Stern SH et al (2002) Use of helical computed tomography for the assessment of acetabular osteolysis after total hip arthroplasty. J Bone Joint Surg Am 84-A:609-614

33. Cahir JG, Toms AP, Marshall TJ et a (2007) CT and MRI of hip arthroplasty. Clin Radiol 62:1163-1171

34. White LM, Kim JK, Mehta M et al (2000) Complications of total hip arthroplasty: MR imaging - initial experience. Radiology 215:254-262

35. Walde TA, Weiland DE, Leung SB et al (2005) Comparison of CT, MRI, and radiographs in assessing pelvic osteolysis: a cadaveric study. Clin Orthop Relat Res 437:138-144

36. Chang JD, Lee SS, Hur M et al (2005) Revision total hip arthroplasty in hip joints with metallosis: a single-center ex perience with 31 cases. J Arthroplasty 20:568-573

37. Heffernan EJ, Alkubaidan FO, Nielsen TO et al (2008) The imaging appearances of metallosis. Skeletal Radiol 37:59-

38. Berry DJ, Harmsen WS, Cabanela ME et al (2002) Twenty-five-year survivorship of two thousand consecutive primary Charnley total hip replacements: fac tors affecting survivorship of acetabular and femoral components. J Bone Joint Surg Am 84-A:171-177

39. Fisher J, Jin Z, Tipper J et al (2006) Tribology of alternative bearings. Clin Orthop Relat Res 453:25-34

40. Lhotka C, Szekeres T, Steffan I et al (2003) Four-year study of cobalt and chromium blood levels in patients managed with two different metal-on-metal total hip replacements. J Orthop Res 21:189-195

41. Willert HG, Buchhorn GH, Fayyazi A et al (2005) Metal-on-metal bearings and hypersensitivity in patients with artificial hip joints. A clinical and histomorphological study. J Bone Joint Surg Am 87:28-36

42. Pandit $\mathrm{H}$, Glyn-Jones $\mathrm{S}$, Mclardy-Smith P et al (2008) Pseudotumours associated with metal-on-metal hip resurfacings. J Bone Joint Surg Br 90:847-851

43. Campbell P, Ebramzadeh E, Nelson $S$ et al (2010) Histological features of pseudotumor-like tissues from metalon-metal hips. Clin Orthop Relat Res 468:2321-2327

44. Chang EY, Mcanally JL, Van Horne JR et al (2012) Metal-on-metal total hip arthroplasty: do symptoms correlate with MR imaging findings? Radiology 265:848-857
45. Van Der Weegen W, Brakel K, Horn RJ et al (2014) Comparison of different pseudotumor grading systems in a single cohort of metal-on-metal hip arthroplasty patients. Skeletal Radiol 43:149155

46. Anderson $\mathrm{H}$, Toms AP, Cahir JG et al (2011) Grading the severity of soft tissue changes associated with metal-onmetal hip replacements: reliability of an MR grading system. Skeletal Radiol 40:303-307

47. Clohisy JC, Calvert G, Tull F et al (2004) Reasons for revision hip surgery: a retrospective review. Clin Orthop Relat Res 428:188-192

48. Cyteval C, Hamm V, Sarrabere MP et al (2002) Painful infection at the site of hip prosthesis: $\mathrm{CT}$ imaging. Radiology 224:477-483

49. Potter H, Foo L, Nestor B (2005) What is the role of magnetic resonance imaging in the evaluation of total hip arthroplasty? HSS J 1:89-93

50. Potter HG, Foo LF (2006) Magnetic resonance imaging of joint arthroplasty. Orthop Clin North Am 37:361-373, vi-

51. Singh JA, Jensen MR, Harmsen SW et al (2013) Are gender, comorbidity, and obesity risk factors for postoperative periprosthetic fractures after primary total hip arthroplasty? J Arthroplasty 28:126-131.e1-e2

52. Hoffmann A, Pfirrmann CWA (2012) The hip abductors at MR imaging. Eur J Radiol 81:3755-3762

53. Pfirrmann CW, Notzli HP, Dora C et al (2005) Abductor tendons and muscles assessed at MR imaging after total hip arthroplasty in asymptomatic and symptomatic patients. Radiology 235:969976

54. Trousdale RT, Cabanela ME, Berry DJ (1995) Anterior iliopsoas impingement after total hip arthroplasty. J Arthroplasty 10:546-549

55. Neal B, Gray H, Macmahon S et al (2002) Incidence of heterotopic bone formation after major hip surgery. ANZ J Surg 72:808-821

56. Bedi A, Zbeda RM, Bueno VF et al (2012) The incidence of heterotopic ossification after hip arthroscopy. Am J Sports Med 40:854-863

57. Seegenschmiedt MH, Goldmann AR, Martus P et al (1993) Prophylactic radiation therapy for prevention of heterotopic ossification after hip arthroplasty: results in 141 high-risk hips. Radiology $188: 257-264$

58. Brooker AF, Bowerman JW, Robinson RA et al (1973) Ectopic ossification following total hip replacement. Incidence and a method of classification. J Bone Joint Surg Am 55:1629-1632

59. Ledermann HP, Schweitzer ME, Morrison WB (2002) Pelvic heterotopic ossification: MR imaging characteristics. Radiology 222:189-195 


\section{CME-Fragebogen}

Bitte beachten Sie:

- Teilnahme nur online unter: springermedizin.de/eAkademie

- Die Frage-Antwort-Kombinationen werden online individuell zusammengestellt.

- Es ist immer nur eine Antwort möglich

Wie kommen Streifenartefakte in der Computertomographie (CT) zustande?

$\square$ Monochromatische Röntgenstrahlen bei $140 \mathrm{keV}$

$\square$ Aufhärtung der Röntgenstrahlung

$\square$ „Photon starvation"

$\square$ Streustrahlen

$\square$ Monochromatische Röntgenstrahlen bei $80 \mathrm{keV}$

? Welches Artefakt wird nicht durch Metall in der Magnetresonanztomographie (MRT) verursacht?

$\square$ Distortion-Artefakt

$\square$ "Signal-loss"-Artefakt

$\square$ „Pile-up"-Artefakt

$\square$ "Through-plane"-Artefakt

$\square$,Scatter"-Artefakt

Auf welchem Prinzip basiert die Metallartefaktreduktion mittels Dual-Energy-CT?

$\square$ Einsatz von SWIFT (,sweep imaging with Fourier transformation")

$\square$ Durch den monochromatischen Datensatz können Aufhärtungsartefakte vermieden oder reduziert werden.

$\square$ Iterative Rekonstruktionsalgorithmen

$\square$ Bildrekonstruktion mit unterschiedlichen $\mathrm{mAs}$

$\square$ Simulation eines virtuellen polychromatischen Datensatzes

Welche der folgenden Methoden kann am besten „Through-plane“-Artefakte in der MRT reduzieren.

$\square$ Untersuchung in 3,0 T statt 1,5 T

$\square$,View-angle tilting"

$\square$ Sequenz mit SEMAC

$\square$ Einsatz einer Gradientenechosequenz

Reduzieren der Empfängerbandbreite
Periprothetische Osteolysen ...

entstehen durch Polyethylenabriebpartikel.

$\square$ entstehen durch zu wenig körperliche Aktivität.

$\square$ kommen nur bei nichtzementierten Prothesen vor.

$\square$ nehmen kein Kontrastmittel auf.

$\square$ sind mit Infekten assoziiert.

Ein Patient mit schmerzhafter MetallMetall-Hüftprothese, die vor 5 Jahren implantiert wurde, wird mittels MRT untersucht. Sie finden eine $6 \mathrm{~cm}$ große Flüssigkeitskollektion mit dickem T2-hypointensem Randwall. Was ist die wahrscheinlichste Diagnose?

$\square$ Abszess

$\square$ Pseudotumor

$\square$ Serom

$\square$ Altes Hämatom

$\square$ Osteolyse

Bei welchem operativen Zugangsweg für eine Hüfttotalprothese wird der M. gluteus medius am meisten geschädigt?

$\square$ Anteriorer Zugang

$\square$ Anterolateraler Zugang

$\square$ Lateraler Zugang

Medialer Zugang

Posteriorer Zugang

Wie kommt das anteriore Iliopsoassehnen-Impingement nach Hüftprothese am ehesten zustande?

$\square$ Narbige Veränderungen nach anteriorem Operationszugang

$\square$ Raumfordernde Wirkung eines Pseudotumors

Reaktive Synovitis durch Abriebpartikel
Hervorstehender anteriorer Rand der Acetabulumkomponente

Scharfe Knochenkante am anterioren Acetabulum

Welche der folgenden Pathologien ist bei einem Patienten mit schmerzhafter Metall-Metall-Prothese am ehesten für seine Schmerzen verantwortlich?

$\square$ Pseudotumor

$\square$ Knochenmarködem

$\square$ Narbe im anterolateralen Operationszugang

$\square$ Gelenkerguss

$\square$ Kleine reife heterotope Ossifikation über dem Trochanter major

Ein Patient, welcher vor 6 Monaten eine nichtzementierte Hüftprothese erhalten hat, wird mittels CT wegen schmerzhafter Hüftprothese abgeklärt. Es gibt eine postoperative Voruntersuchung. Was erklärt seine Schmerzen am ehesten?

$\square$ Neuer feiner Lysesaum $<2$ mm

$\square$ Neues Einsinken der Schaftprothese um $8 \mathrm{~mm}$

$\square$ Zunehmende Osteopenie an der Trochanter-major-Region

$\square$ Kleine heterotope Ossifikationen

$\square$ Neue bandförmige Sklerosezone im Acetabulumdach

Diese zertifizierte Fortbildung ist 12 Monate auf springermedizin.de/ eAkademie verfügbar.

Dort erfahren Sie auch den genauen Teilnahmeschluss. Nach Ablauf des Zertifizierungszeitraums können Sie diese Fortbildung und den Fragebogen weitere 24 Monate nutzen. 